\title{
Ho LOOP SHAPING CONTROL DESIGN OF THE ROTATIONAL VELOCITY OF A HYDRAULIC MOTOR
}

\author{
Hasan H. Ali ${ }^{*}$, Salwan Obaid Waheed Khafaji ${ }^{2}$, Fawaz F. Al-Bakri ${ }^{3}$ \\ ${ }^{1}$ Directorate of Reconstruction and Projects; Ministry of Higher Education and Scientific Research, Baghdad, Iraq \\ 2University of Babylon, College of Engineering, Mechanical Department, Babylon, Iraq. \\ ${ }^{3}$ Technical Institute of Babylon; Al-Furat Al-Awsat Technical University (ATU), Iraq. \\ *corresponding author: Hasan H. Ali \\ Email: hha2mf@mail.missouri.edu
}

\begin{abstract}
In this work, a hydraulic system in which the hydraulic motor velocity is controlled using a variable speed pump. A comprehensive dynamic model was created and the performance and stability of the open loop system were assessed. Then, PID and loop shaping H-infinity controllers were designed with the aim of improving the system performance. The multiplicative uncertainty, that is associated with uncertain parameters in the speed controlled velocity control system, was studied. Two parameters were introduced in the uncertainty analysis; the leakage coefficient and the viscous friction coefficient. The requirements of the system performance were defined in the frequency domain that was used to determine the robustness of the closed-loop velocity control system. The results showed that the proposed open loop hydraulic system is stable. It was also shown that using feedback control greatly improves the system performance. The performance of the nominal system, based on the obtained results, with Ho controller was found to be better than that of the system with PID controller. It was found that the Ho controller has the advantages of having robust performance by considering the uncertainty in addition to not having an integral control as in the PID control system. In addition, it was found that the system with the PID controller did not achieve robust performance.
\end{abstract}

Keywords: Pump; Hydraulic motor; Variable speed; Velocity control; Robust control; Uncertainty.

\section{Introduction}

Hydraulic motors are extensively used in engineering applications, such as off-highway airplanes and vehicles. They used along with pumps and some accessories to form what is called hydrostatic transmission. They convert the fluid power which is generated by pumps in the place it is generated into mechanical power in the place it is consumed. Hydrostatic transmission is characterized by its high-power density and high efficiency. Hydraulic motors are usually required to deliver variable amount of power based on the system requirements such as position, velocity, torque, etc.

The requirement of delivering variable power is attained by controlling the flow rate of the hydraulic fluid delivered by the pump. Variable flow control techniques are used in fluid power systems such as displacement control systems [1-3], speedcontrolled systems [4-6], and valve control systems [7-9]. A comparison of those techniques can be found in [10]. In [11], a model was created for the flow and torque of hydraulic motor and efficiency maps were plotted. The model was validated using experimental data. The results showed that the efficiency of a hydraulic motor can exceed $94 \%$.

Different control methods have been used to control the motion of hydraulic actuators. A cascade controller was considered in [12] to control the position of a hydraulic motor. A dynamic model was created for the system which was validated using experimental data. In [13], sliding mode control was used to control the motion of a hydraulic actuator. In [14], H-infinity controller, two degrees of freedom controller (2DOF), and PID controller were designed to control the velocity of a linear hydraulic actuator. Inlet throttled pump was used for the flow control purposes. The closed loop performance of the system was compared with that of the open-loop system which showed improvement in the system performance especially when 2DOF controller were used. The uncertainty of the inlet metered velocity control system was studied in [15] which showed that although the performance of the system with $\mathrm{H}$ infinity and PID controllers is almost the same, only $\mathrm{H}$-infinity satisfies the requirements of robustness. 
A robust quantitative feedback theory based controller for controlling a force of a hydraulic actuator was designed in [16] with the goal of improving the disturbance rejection performance, and stability of the system. In [17], two degrees of freedom controller was designed to control the position of a hydraulic actuator. A robust PID and $\mathrm{H}$ infinity controllers were designed in [18] to control the velocity of a hydraulic linear actuator subjected to parametric uncertainty. A variable speed pump was used as the variable flow source. A model was created and open loop and closed loop performance was studied in the frequency domain and time domain. A simplified model of the hydraulic motor is given in [19].

In this paper, a new comprehensive model for a hydrostatic transmission that utilizes a hydraulic motor was created. A speed controlled fixed displacement pump was used to deliver variable flow to the hydraulic motor. The variable flow supplied by the variable speed pump provides a precise control over the motion of the hydraulic motor. Speed controlled systems have lower cost than displacement controlled systems and better performance than valve controlled systems. Based on the dynamic model of the system, PID and $\mathrm{H}$ infinity controllers were designed for the system subjected to parametric uncertainty and the performance of the system with both controllers was compared with that of the open loop system. Lastly, robustness of the closed loop system with the PID and $\mathrm{H}$-infinity controllers was studied.

\section{System Model}

Figure. 1 shows the components of the system. The system consists of a fixed-displacement variable speed pump to control the motion of a fixeddisplacement hydraulic motor. In this figure, $J, b$, and $k_{t}$, refers to the mass, spring rate, and viscous drag coefficient for the load of the load to be moved by the motor which is represented by a mass-springdamper system respectively. The symbol $T$ refers to a load disturbance torque. The volumetric displacement of the hydraulic motor is given by the symbol Dm. The hydraulic fluid flows into and out from the hydraulic motor at rates $Q_{A}$ and $Q_{B}$ respectively. The volumetric flow rate of hydraulic fluid flows into the motor is controlled by the output flow rate of the fixed-displacement variable speed. This pump is typically operated at a fixed displacement given by $D_{p}$. The motion of the hydraulic motor is controlled by altering the flow rate supplied by the pump in magnitude and direction. The pump variable flow is achieved by changing the magnitude and direction of the pump rotational speed.

In Fig. 1, $K$ represents the flow coefficient of the orifice flow passages of the circuit which exist inherently within the hydraulic system.
The charge pump circuit is used to generate the pressurized low-pressure side of the hydraulic control system and it is sometimes used as an auxiliary source of pressure for the variable-speed pump control (VSPC). In addition, the charge pump is used along with a relief valve to provide makeup flow to the inlet of the main pump. The check valves are used for the purpose of blocking side A from the charge circuit in addition to opening side $B$ to the charge circuit. This guarantees that side B is always maintained at a constant pressure and prevents cavitation phenomenon when fast alterations of the pump speed occurs [19].

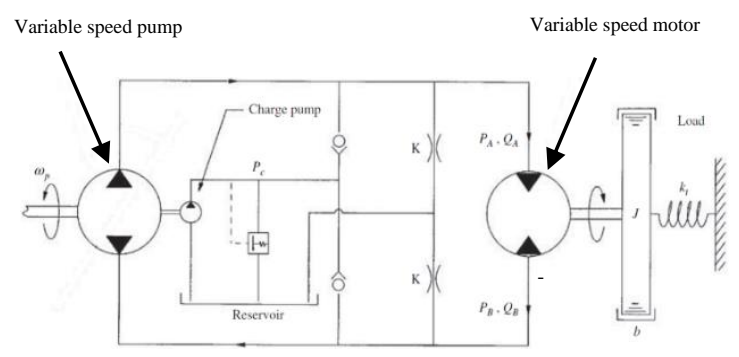

Figure. 1 The hydraulic circuit

\section{Model, Uncertainty, and Control}

The governing equations of the hydraulic control system under consideration are the equation of motion and the equation of the pressure rise rate. The equation of motion may be written as:

$$
J \hat{\theta}_{m}^{*}+b \dot{\theta}_{m}+K_{t} \theta_{m}=\eta_{a f} D_{m} P_{d}-T
$$

where $\theta_{m}$ is the angular displacement of the hydraulic motor and Pd is the difference in pressure across the hydraulic motor $\left(P_{d}=P_{A}-P_{B}\right)$. At steady state working conditions, $\theta_{m}=\dot{\theta}_{m}=\dot{\theta}_{m}=0, T=0$ and $P_{d}=0$, then, $T_{o}=0$. Also, the effective spring is neglected typically in the velocity control systems [10], i.e., $\mathrm{K}_{\mathrm{t}}=0$. Therefore, Eqn. 1 becomes,

$$
J \omega_{m}+b \omega_{m}=\eta_{a} P_{d} D_{m}-T
$$

where the $\dot{\omega}$ is the motor angular acceleration. The equation of the pressure rise rate may be written as:

$$
\dot{P}_{d}=\frac{\beta}{V_{o}+D_{m} \theta_{m}}\left(Q_{p}-K P_{d}-D_{m} \omega_{m}\right)
$$

where $Q_{p}$ is the supplied volumetric flow by the pump which is defined as, $Q_{p}=D_{p} \omega_{p}$. The coefficient of leakage, $K$, was supposed to be a constant. It is suggested by the literature that a sensible value for this coefficient is $K=\frac{Q_{\max }}{10 P_{\max }}$ [14].

Eqn. (3) can be linearized using the following conditions at their nominal values as:

$$
\theta_{m 0}=\omega_{m 0}=Q_{p 0}=P_{d 0}=0 .
$$


The resulted linearized equation for Eqn. (3) is,

$$
\dot{P}_{d}=\frac{\beta}{V_{o}}\left(Q_{p}-K P_{d}-D_{m} \omega_{m}\right)
$$

Substituting for $Q_{p}$ gives:

$$
\dot{P}_{d}=\frac{\beta}{V_{o}}\left(D_{p} \omega_{p}-K P_{d}-D_{m} \omega_{m}\right)
$$

For generalizing the model and reducing the number of variables, all the parameters in Eqs. (2) and (5) were normalized about some reference conditions. Using the following reference conditions:

$$
\omega=\widehat{\omega} \omega_{r}, P_{A}=\hat{P}_{d} P_{d r} \text { and } t=\hat{t} \tau \text {. }
$$

Equation (2) is written as:

$$
J \frac{d\left(\hat{\omega}_{m} \omega_{m r}\right)}{d(\hat{t} \tau)}+b \widehat{\omega}_{m} \omega_{m r}=\eta_{a f} \hat{P}_{d} P_{d r} D_{m}-T
$$

Dividing Eq. (6) by $P_{d r} D_{m} \eta_{a f}$ gives

$$
\hat{J} \widehat{\omega}_{m}+\widehat{b} \widehat{\omega}_{m}=\hat{P}_{A}-\hat{T}
$$

Where

$$
\left\{\begin{array}{l}
\hat{J}=\frac{J \omega_{r}}{P_{d r} D_{m} \tau \eta_{a f}} \\
\hat{b}=\frac{b \omega_{m r}}{P_{d r} D_{m} \eta_{a f}} \\
\hat{T}=\frac{T}{P_{d r} D_{m} \eta_{a f}}
\end{array}\right\}
$$

Correspondingly, based on the above conditions, Eq. (5) may be updated as;

$$
\frac{d\left(\hat{P}_{d} P_{d r}\right)}{d(\hat{t} \tau)}=\frac{\beta}{V_{o}}\left(D_{p} \omega_{p} \widehat{\omega}_{p}-K \hat{P}_{d} P_{d r}-D_{m} \omega_{m} \widehat{\omega}_{m}\right)
$$

Multiplying Eq. (9) by ? $/ P_{d r}$ gives:

$$
\stackrel{\hat{P}}{d}_{d}=\frac{\tau \beta}{V_{0} P_{A r}} D_{p} \omega_{p r} \hat{\omega}_{p}-\frac{\tau \beta}{V_{0}} K \hat{P}_{d}-\frac{\tau \beta}{V_{0} P_{d r}} D_{m} \omega_{m r} \hat{\omega}_{m}
$$

Let

$$
\tau=\frac{V_{o}}{\beta \mathrm{K}}
$$

then, that the equation of the pressure rise can be written in the non-dimensional as,

$$
\hat{P}_{d}=\pi_{1} \widehat{\omega}_{p}-\hat{P}_{d}-\pi_{2} \widehat{\omega}_{m} .
$$

Figure 1 Electromagnetic Actuator Schematic

In figure (1), $\mathrm{m}$ is the moving piston mass and $K$ is the spring stiffness. $F_{f r}$ and $F_{\text {spring }}$ are forces that are generated due to friction and spring, respectively.
The variables $s(t), v(t)$, and $i(t)$ are the actuator position, velocity, and, current, respectively, which are considered as the system states: $[s(t) v(t) i(t)]=$ $\left[\begin{array}{lll}x_{1} & x_{2} & x_{3}\end{array}\right]$. Moreover, the coil voltage, $V$ is considered as the system input: $[V]=[u]$. A full state-space representation (SSR) of the electromagnetic actuator system can be ob $\pi_{1}$ and $\pi_{2}$, the non-dimensional groups, in Eq. (11) are defined as,

$$
\left\{\begin{array}{c}
\pi_{1}=\frac{D_{p} \omega_{p r}}{P_{d r} K} \\
\pi_{2}=\frac{D_{m} \omega_{m r}}{P_{d r} K}
\end{array}\right\}
$$

The values of the quantities in Eqs. (7) and (11) are given in Table 1 below,

Table 1: Dimensionless quantities in Eqs. (7) and (11).

\begin{tabular}{|c|c|}
\hline Quantity & value \\
\hline$\hat{b}$ & 0.15 \\
\hline$\hat{J}$ & 5.84 \\
\hline$\widehat{T}$ & 0.82 \\
\hline$\pi_{1}$ & 10 \\
\hline$\pi_{2}$ & 8.89 \\
\hline
\end{tabular}

\section{Stability Analysis}

The equations of the system dynamics, Eqs. (9) and (11), can be introduced in state-space matrix form, i.e.,

$$
\begin{aligned}
& \dot{x}=A \boldsymbol{x}+B \boldsymbol{u} \text { and } y=C \boldsymbol{x}+D \boldsymbol{u} \\
& \text { Let } \boldsymbol{x}=\left[\widehat{P}_{d} \widehat{\omega}_{m}\right]^{T}, \boldsymbol{u}=\left[\widehat{\omega}_{p} \hat{T}\right]^{T} \text { and } \boldsymbol{y}=\widehat{\omega}_{m} .
\end{aligned}
$$

By inspecting Eqs. (8) and (10), the matrices in the above representation can be given by,

$$
A=\left[\begin{array}{cc}
-1 & -\pi_{2} \\
\frac{1}{\hat{J}} & -\frac{\hat{b}}{\hat{J}}
\end{array}\right], B=\left[\begin{array}{cc}
\pi_{1} & 0 \\
0 & -\frac{1}{\hat{J}}
\end{array}\right], C=\left[\begin{array}{ll}
0 & 1
\end{array}\right], D=0 .
$$

For analysis of stability, the characteristic equation, then, $\operatorname{det}(s I-A)=\hat{J} s^{2}+(\hat{J}+\hat{b}) s+\left(\hat{b}+\pi_{2}\right)=0$.

Based on Routh-Hurwitz stability criterion, the characteristic equation coefficients are:

$$
\left\{\begin{array}{c}
a_{o}=\hat{J} \\
a_{1}=(\hat{J}+\hat{b}) \\
a_{2}=\left(\hat{b}+\pi_{2}\right)
\end{array}\right\}
$$

It can be seen from Eq. (13) that none of $a_{0}, a_{1}$, and $a_{2}$ can be negative for real life applications which indicates, according to Routh-Hurwitz stability criterion, that the open loop system will be stable.

The transfer function of the system from the reference input to the actual output $(G)$ and from the 
disturbance torque to the actual output $\left(G_{T}\right)$ are given in Eq. (14) and Eq. (15) respectively

$$
\begin{aligned}
& G=\frac{\widehat{\omega}_{R}}{\widehat{\omega}_{p}}=\frac{\pi 1}{\hat{J} s^{2}+(\hat{J}+\hat{b}) s+\left(\hat{b}+\pi_{2}\right)} \\
& G_{T}=\frac{\hat{\omega}_{T}}{\widehat{T}}=\frac{-(s+1)}{\hat{J} s^{2}+(\hat{J}+\hat{b}) s+\left(\hat{b}+\pi_{2}\right)}
\end{aligned}
$$

The next stage is to define the system's uncertainty ant its modeling.

The fluctuation of the parameters considered in this work throughout a known range generates a set of perturbed plants.

This set of plants is used to calculate the multiplicative uncertainty, which would be the relative error.

The multiplicative uncertainty is defined as the difference between the frequency response and nominal plants' frequency responses divided by the nominal plant's frequency response. Equations (16) and (17), respectively, give the multiplicative uncertainty, $l_{I}$, and its rational weight, $w_{I}$.

$$
\begin{aligned}
& l_{I}(\omega)=\max _{G_{\text {pert }} \in \Pi}\left|\frac{G_{\text {pert }}(j \omega)-G_{0}(j \omega)}{G_{0}(j \omega)}\right| \\
& w_{I}(j \omega) \geq l_{I}(\omega), \forall \omega
\end{aligned}
$$

Where $G_{0}(j \omega)$ and $G_{\text {pert }}(j \omega)$ are the nominal and perturbed plant, respectively. The nominal plant, $G_{0}$. was obtained by calculating $G(j \omega)$ using the nominal parameters listed in Table 1 . By evaluating $G_{0}(j \omega)$ with changing plant parameters, the class of perturbed plants utilized to construct $G_{\text {pert }}(j \omega)$ was identified. In the uncertainty analysis, two parameters are taken into account. Those two parameters were the viscous damping coefficient and the leakage coefficient. The range of variation was considered to be within $\pm 5 \%$. For all potential plant perturbations over all frequencies, Equation (17) requires that the uncertainty weight transfer function, $w_{I}$, must be bigger than the maximum error, $l_{I}$. (the worst-case scenario). The upper bound limit on the multiplicative error in Eq. (16) was calculated numerically by determining the nominal plant, $G_{0}(\omega)$, and plant perturbations, $G_{\text {pert }}(j \omega)$, using a grid of plant parameter values in the provided ranges. Then the multiplicative error (the part of Eq. (16) bounded by the absolute value bars) was determined for a range of frequencies given the perturbed plants and the nominal plant (see Fig. 5 introduced later in the Results and Discussion section). Then the maximum value of the frequency responses magnitude in Fig. 5 was calculated by using Eq. (16).

To obtain a bounding transfer function, the uncertainty weight transfer function (UWTF) was created numerically by a transfer function fitting to be an approximation of the frequency response for the maximum values of the multiplicative uncertainty (shown in Fig. 5 in red). UWTF, $w_{I}$, presented by the upper bound on the uncertainty as in Eq. (17) can be given by Eq. (18).

$$
w_{I}=\frac{s^{2}+6.6 s+10.89}{33 s^{2}+145.2 s+159.7}
$$

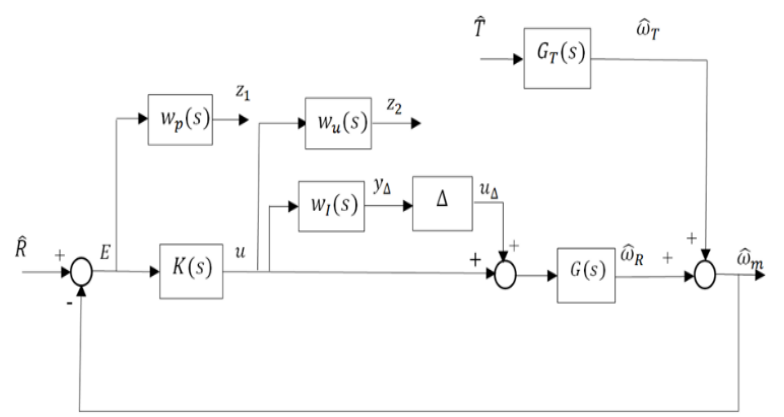

The block diagram, with the control effort weight, $w_{u}$, performance weight, $w_{p}$, and the multiplicative uncertainty weight, $w_{I}$, and the transfer functions is shown in Fig. 2.

Figure 2: The block diagram of the feedback control system with the weights

In Fig. 2, two signals, $z_{1}$ and $z_{2}$, refer to the control system's performance metrics, the weighed error and weighted control effort, respectively. The sensitivity transfer function of the feedback control system in Fig. 2 is the transfer function that connects the reference input, $R$, to the error, $E$. In the frequency domain, the system's performance requirements can be stated in terms of the required bandwidth of the sensitivity transfer function, low and high frequency error levels. Eq. (19) is used to calculate the performance weight for performance analysis, which is the inverse form of the upper bound on the frequency response of the sensitivity transfer function, $w_{p}$, [20].

$$
w_{p}(s)=\frac{\frac{s}{M}+\widehat{\omega}_{b}}{s+a \widehat{\omega}_{b}}
$$

The non-dimensional magnitudes of the constants in Eq. (19) in this work were chosen to be as follows; the bandwidth frequency is $\widehat{\omega}_{b}=0.2$, the high frequency error value is $M=6$, and the low frequency error is $a=0.05$.

In Fig 2, the system's performance can be further defined in terms of the control effort, u. Because of the model's nondimensional nature, it's best to keep the control effort under one. As a result, the control effort weight, $\mathrm{w} u$, illustrated in Fig. 2, is selected to be one, which is typical value for the nondimensional system with input normalized around its maximum value. With the two weights selected, the performance outputs can be examined in the frequency domain in relation to the external inputs. 
In this paper, two feedback controllers are considered. The PID controller was designed based on the nominal plant along with the auto tuning that was used to determine the gains. The proportional, integral, and derivative gains of the PID controller were $3.22,1.7$, and 1.37 respectively. It is worthy to note that the derivative term in this equation is zero. An $\mathrm{H}_{\infty}$ control design was also considered.

The controller, $\mathrm{K}(\mathrm{s})$, is calculated by obtaining a transfer function that achieve a minimum value for the $\mathrm{H}_{\infty}$ norm for the matrix of the transfer function developed from Fig. 2 by connecting the external inputs $(\widehat{\mathrm{R}}$ and $\widehat{\mathrm{T}})$ to the outputs $\mathrm{z}_{1}$ and $\mathrm{z}_{2}$ while ignoring the uncertainty (letting $\Delta=0$ ).

The controller, $K(s)$, is obtained by finding a controller transfer function that minimizes the norm of the transfer function matrix formed from Fig. 2 by relating the external inputs $(\hat{R}$ and $\hat{T})$ to the outputs $z_{1}$ and $z_{2}$ while ignoring the uncertainty (letting $\Delta=0$ ). For this to be done, Equation (20) should be satisfied [14].

$$
\left\{\begin{array}{c}
\left\|S G_{d} w_{p}(j \omega)\right\|_{\infty}<1 \\
\left\|S w_{p}(j \omega)\right\|_{\infty}<1 \\
\left\|S K w_{u}(j \omega)\right\|_{\infty}<1 \\
\left\|S K G_{d} w_{u}(j \omega)\right\|_{\infty}<1
\end{array}\right\}
$$

Using a typical $\mathrm{H}_{\infty}$ optimization controller synthesis methodology, the transfer function of $\mathrm{H}_{\infty}$ controller was produced using the Matlab $^{\circledR}$ function hinfsyn.m as shown in Eq. (21).

$$
\mathrm{K}(s)=\frac{2.98 \mathrm{~s}^{2}+3.054 \mathrm{~s}+4.604}{s^{3}+4.404 s^{2}+5.319 s+0.05275}
$$

The system in Fig. 2 is then turned into a generalized plant transfer function, which aids in the examination of stability and performance using the performance weights and uncertainty model. From Fig. 2, $y_{\Delta}, z_{1}, z_{2}$, and $E$ can be written as shown in Eqs. (22-25).

$$
\begin{aligned}
& y_{\Delta}=w_{I} u \\
& z_{1}=-G_{0} w_{p} u_{\Delta}+w_{p} \hat{R}-G_{T} w_{p} \hat{T}-G_{0} w_{P} u \\
& z_{2}=w_{u} u \\
& E=-G_{0} u_{\Delta}+I \hat{R}-G_{T} \hat{T}-G_{0} u
\end{aligned}
$$

The generalized plant, $P$, presented in Fig. 3 is obtained by,

$$
\left[\begin{array}{c}
y_{\Delta} \\
z \\
E
\end{array}\right]=[P]\left[\begin{array}{l}
u_{\Delta} \\
w \\
u
\end{array}\right]
$$

where $w$ is exogenous inputs and $z$ is the exogenous outputs and can be defined in Eq. (27) and Eq. (28) respectively.

$$
w=\left[\begin{array}{l}
\hat{R} \\
\hat{T}
\end{array}\right]
$$

$$
z=\left[\begin{array}{l}
z_{1} \\
z_{2}
\end{array}\right]
$$

The components of the $P$ matrix, $P_{11}, P_{12}, P_{21}$, and $P_{22}$,as shown in Eq. (29) can be defined in Eqs. (3033).

$$
\begin{aligned}
P= & {\left[\begin{array}{ll}
P_{11} & P_{12} \\
P_{21} & P_{22}
\end{array}\right] } \\
P_{11} & =\left[\begin{array}{ccc}
0 & 0 & 0 \\
-G_{0} w_{P} & w_{p} & -G_{T} w_{p} \\
0 & 0 & 0
\end{array}\right] \\
P_{12} & =\left[\begin{array}{c}
w_{u} \\
-G_{0} w_{P} \\
w_{p}
\end{array}\right] \\
P_{21} & =\left[\begin{array}{lll}
-G_{0} & I & -G_{T}
\end{array}\right] \\
P_{22} & =\left[\begin{array}{ll}
-G_{0}
\end{array}\right]
\end{aligned}
$$

Substituting Eqs. (30-33) into Eq. (29) gives the $P$ matrix as shown in Eq. (34).

$$
P=\left[\begin{array}{cccc}
0 & 0 & 0 & w_{u} \\
-G_{0} w_{P} & w_{p} & -G_{T} w_{p} & -G_{0} w_{P} \\
0 & 0 & 0 & w_{p} \\
-G_{0} & I & -G_{T} & -G_{0}
\end{array}\right]
$$

When the controller design is developed based on both performance and uncertainty, the structured matrix, $\widehat{\Delta}$, is considered [15]. This matrix is defined as shown in Eq. (35).

$$
\widehat{\Delta}=\left[\begin{array}{rr}
\Delta & 0 \\
0 & \Delta_{P}
\end{array}\right]
$$

The two terms, $\Delta$ and $\Delta_{P}$ are the uncertainty of the model and the performance, respectively. From Fig. 4 and Eqs. (17 and 18), $\Delta$ has one input, $y_{\Delta}$, and one output, $u_{\Delta}$, while $\Delta_{P}$ has two inputs $\left(z_{1}\right.$ and $\left.z_{2}\right)$ and two outputs $(\hat{R}$ and $\hat{T})$. In addition, the controller, $K$, has one input and one output, $E$ and $u$, respectively, as shown in Fig. 3. The nominal system matrix, $N$, is related to the plant, $P$, and the controller, $K$, by a lower linear fractional transformations (LFT) given in Eq. (36) [20].

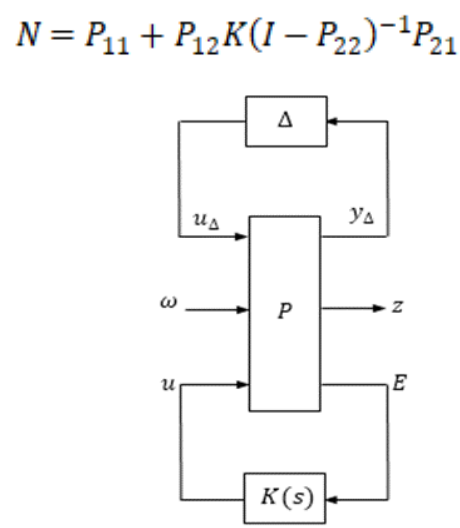

Figure 3: The general control configuration (for controller synthesis) 


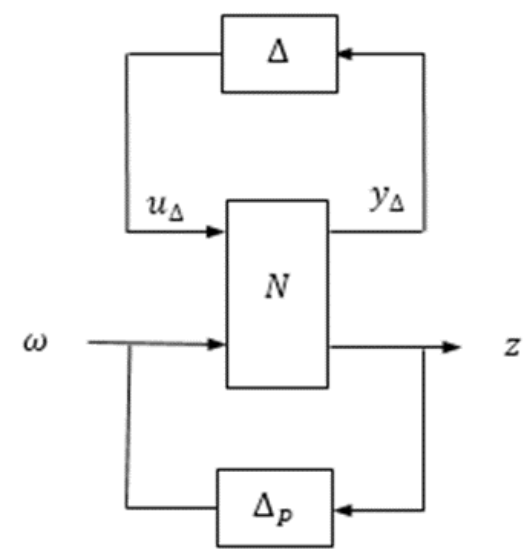

Figure 4: The $N-\triangle$ structure

It can be noticed that $N$, From Fig. 4 , has three inputs $\left(\hat{R}, u_{\Delta}\right.$, and $\left.\hat{F}\right)$ and three outputs $\left(z_{1}\right.$, and $z_{2}$, and $y_{\Delta}$, ) and may be given as shown in Eq. (37).

$$
\left[\begin{array}{l}
y_{\Delta} \\
z_{1} \\
z_{2}
\end{array}\right]=[N]\left[\begin{array}{l}
u_{\Delta} \\
\hat{R} \\
\hat{T}
\end{array}\right]
$$

To study the robustness of the system, $N$ is partitioned into four elements $\left(N_{11}, N_{12}, N_{21}\right.$, and $N_{22}$ ) in a specific way similar to that used for portioning the generalized plant $P$.

\section{Stability and Performance Criteria}

After determining the $N$ matrix, an analysis was carried out to check the nominal and perturbed systems' stability and performance. The system must meet the robust and the nominal stability and performance criteria listed in [20].

Nominal consistency (NS). The nominal stability is attained if and only if the system is stable without taking into account the uncertainties. This signifies that the nominal model's poles are all on the left half plane.

Nominal Performance (NP). If the system is nominally stable and meets the performance requirements without taking into account the uncertainties, the nominal performance is attained. This is possible only if $N_{22}$ infinity norm (specified in [20]) is less than one, as proven in Eq. (38). The model is on the left half of the plane.

$$
N P \Leftrightarrow\left\|N_{22}\right\|_{\infty}<1
$$

Robust Stability (RS). If the system remains stable for all perturbed plants in the uncertainty set, it is considered to be robustly stable. The nominal system is stable if and only if the infinity norm of $N_{11}$ is less than one, as stated in Eq. (39), and less than one.

$$
R S \Leftrightarrow\left\|N_{11}\right\|_{\infty}<1
$$

Robust Performance (RP). If the system achieves the performance requirements for all perturbed plants in the uncertainty set and is nominally stable, it is said to have robust performance.

$$
R P \Leftrightarrow \mu(N, \widehat{\Delta})<1
$$

The maximum structured singular value of $N$ must be smaller than one for this to be true, as stated in Eq. (40).

\section{Results and Discussion}

The hydraulic system that utilizes a hydraulic motor was modelled and the performance of the open loop system was studied. Then, PID and $\mathrm{H}_{\infty}$ controllers were designed to improve the system performance. Matlab Simulink ${ }^{\circledR}$ autotuning was used to determine the PID controller gains while the $\mathrm{H}_{\infty}$ controller was designed to satisfy Eq. (20). The designed $\mathrm{H}_{\infty}$ controller satisfies the conditions specified in Eq. (20) as shown in Figs. 5 and 6. The multiplicative error associated with the velocity control system due to the variation in the design parameters over a frequency range is shown in Fig. 7. It can be seen that with the current range of parameter variation, there is uncertainty of about seven percent at low and medium frequencies and about three percent at high frequencies.

Figure 8 show that both the PID and the $\mathrm{H}_{\infty}$ controllers encounter the nominal stability and robust stability. However, only the $H_{\infty}$ controller meets robust performance and the nominal requirements as shown in Figs. 9 and 10, where maximum value of $\mu_{\Delta}$ is less than 1 with the $H_{\infty}$ controller and greater than 1 with the PID controller.

Next a simulation of the system in Fig. 2 was used to demonstrate the time domain response of the nominal system without uncertainty with each of the two controllers. The simulation was completed with the following inputs, a unit step to the reference, $\hat{R}$, at zero seconds and a 0.8 step to the disturbance torque, $\widehat{T}$, at 10 seconds. Note that the simulation was accomplished by construction a block diagram as in Fig. 2 in Matlab Simulink ${ }^{\circledR}$ and with the $\Delta$ block signal path in Fig. 2 removed so that only the nominal system would be simulated (i.e. without uncertainty). The time response of the velocity of the nominal system with both controllers is shown in Fig. 11. It can be seen that $\mathrm{H}_{\infty}$ controller provides better response than the PID controller in terms of rise time, settling time, percentage overshoot, and disturbance rejection. It can be noted from Fig. 12 that the pump rotational speed required to achieve the desired actuator position is lower in the case closed loop is used (with both PID and $\mathrm{H}_{\infty}$ controllers) which indicates that less power is required. Saturation was used to limit the pump speed from exceeding a dimensionless value of 1 . 


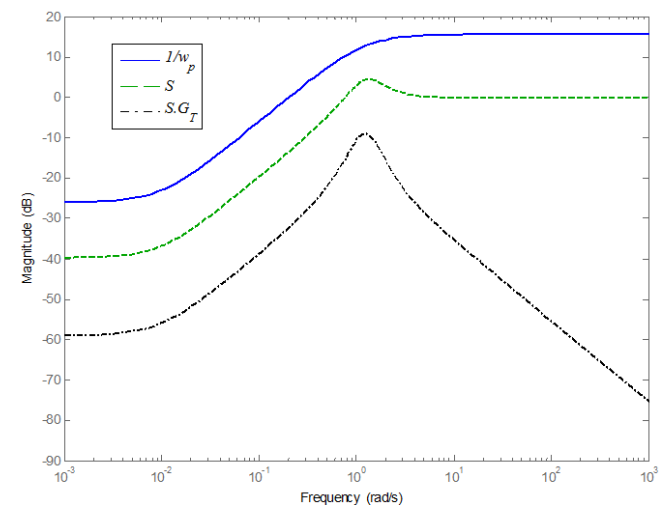

Figure 5: Frequency response of $1 / w_{p}$, S and $S . G_{T}$.

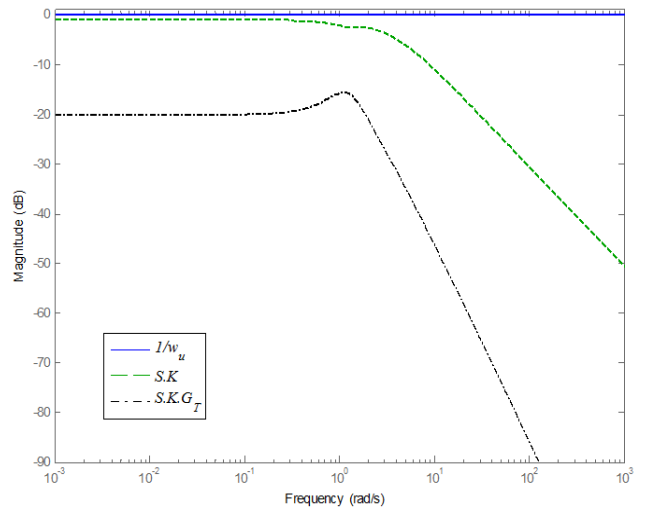

Figure 6: Frequency response of $1 / w_{u}$, S and $S . G_{T}$.

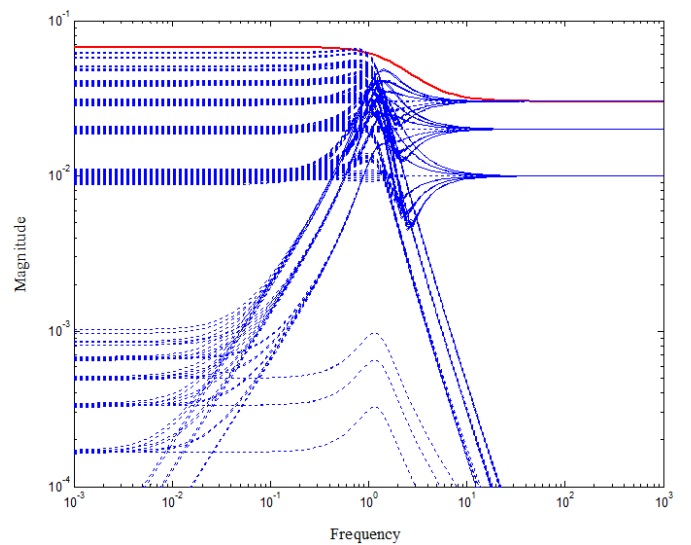

Figure 7: Multiplicative uncertainty transfer function bounding the maximum multiplicative error

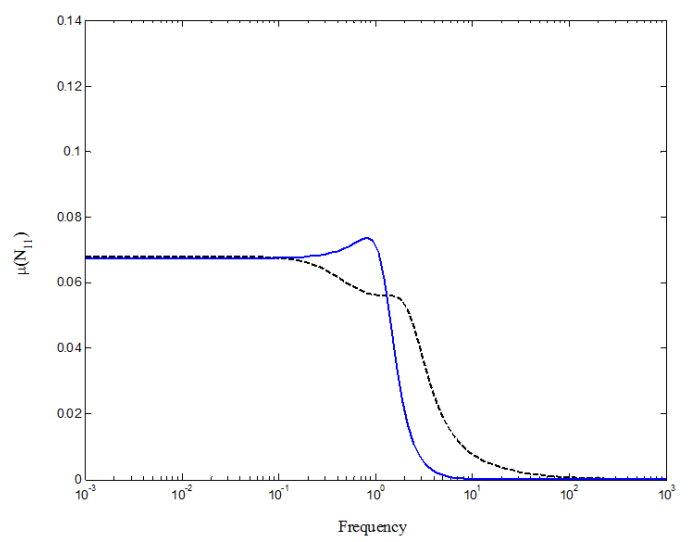

Figure 8.: The robust stability requirement

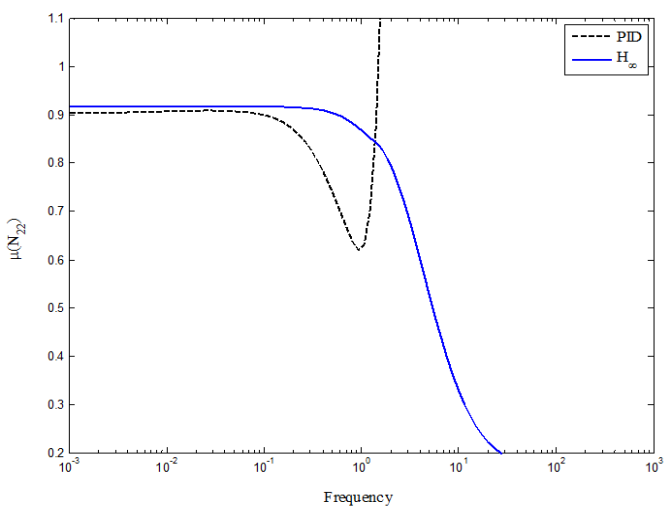

Figure 9: The nominal performance requirement

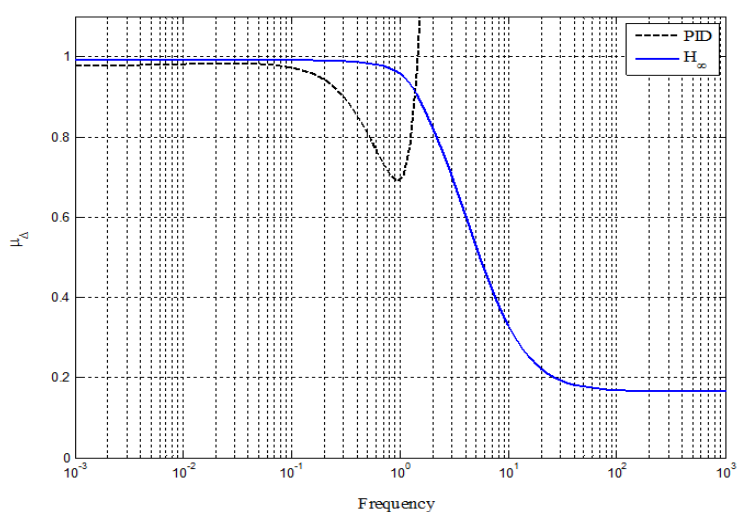

Figure10: The robust performance requirement

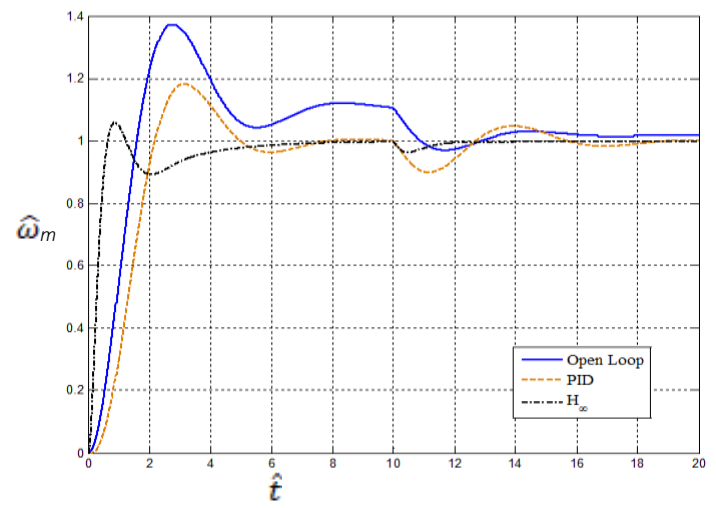

Figure 11: Dimensionless motor rotational speed vs. time

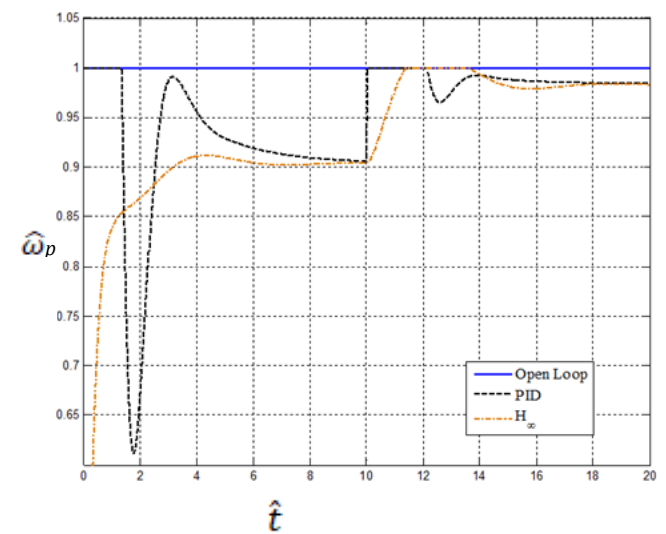

Figure 12: Dimensionless pump rotational speed vs. time 


\section{Conclusions}

A speed controlled hydrostatic transmission utilizes a fixed displacement pump and motor was modelled and investigated. The uncertainty in the system parameters (parametric uncertainty) associated with speed-controlled velocity control system of a hydraulic motor was studied. Stability, performance and robustness to uncertainty in the system parameters of the open loop and the feedback control system with PID and $H_{\infty}$ controllers were studied. The time responses of the system with the PID and $H_{\infty}$ controllers were compared. It can be noted that the closed loop system provides much better response and consumes less power than the open loop system. It can also be concluded that $\mathrm{H}_{\infty}$ controller provides better performance than the PID controller in terms of rising time, settling time, percentage overshoot, and disturbance rejection. While both controllers meet the requirements for nominal stability and robust stability, only the $H_{\infty}$ controller meets the nominal and robust performance requirement. In addition, the implementation of PID controller would likely require some additional complexity because of practical matters like the measures needed to eliminate integrator windup. In addition, the $H_{\infty}$ controller has no pure integrator, and thus it does not require an anti-windup scheme which is considered another advantage of the $H_{\infty}$ controller.

\section{References}

[1] Manring, N.D. and Johnson, R.E. "Modeling and Designing of a variable Displacement Open-Loop Pump." Journal of Dynamic Systems, Measurement, and Control, Vol. 118, (1996) pp.267-271.

[2] Manring, N.D. and Luecke, G.R. "Modeling and Designing a Hydrostatic Transmission with a Fixed-Displacement Motor". Journal of Dynamic Systems, Measurement, and Control, Vol. 120(1998), pp.45-49.

[3] Wang, S. "Improving the Volumetric Efficiency of the Axial Piston Pump". Journal of Mechanical Design, Vol. 134, (2013), pp. 111001/1111001/7.

[4] Hu D, Ding S, Zhu H, Xu B, Yang H (1995) Velocitytracking control of the variable-speed controlled hydraulic system: using compound algorithm of PD \& feedforward-feedback control, pp. 11091115. ttps://doi.org/10.1109/ICMTMA.2011.846

[5] Calıskan H, Balkan T, Platin BE (2009) Hydraulic position control system with variable speed pump, ASME Paper No. DSCC2009-2693.

[6] Mandal, S.K., Singh, A.K., Verma, Y. et al. Performance Investigation of Hydrostatic Transmission System as a Function of Pump Speed and Load Torque. J. Inst. Eng. India Ser. C 93, 187-193

(2012). https://doi.org/10.1007/s40032-012-0022-4.

[7] Zhang, Qin., 1999, "Hydraulic Linear Actuator Velocity Control Using a Feedforward-plus-PID Control," International Journal of Flexible
Automation and Integrated Manufacturing, Vol.7, No. 3, pp. 277-292.

[8] Ali HH, Wisch JK, Fales RC, Manring ND (2019) Efficiency of a fixed displacement pump with flow control using an inlet metering valve. J Dyn Syst Meas Control 141:1-14. https://doi.org/10.1115/1.4041606.

[9] Yuan C, Pan M, Plummer A (2018) A review of switched inertance hydraulic converter technology. In: Proceedings of the BATH/ASME 2018 symposium on fluid power and motion control. Bath, UK. September 12-14, 2018. V001T01A013. https://doi.org/10.1115/FPMC2018-8829.

[10] Ali, H.H., Fales, R.C. A review of flow control methods. Int. J. Dynam. Control (2021). https://doi.org/10.1007/s40435-020-00730-y.

[11] Manring ND (2016) Mapping the efficiency for a hydrostatic transmission. J Dyn Syst Meas Control 138:1-8. https://doi.org/10.1115/1.4032289

[12] P. M. La Hera, U. Mettin, S. Westerberg and A. S. Shiriaev, "Modeling and control of hydraulic rotary actuators used in forestry cranes," 2009 IEEE International Conference on Robotics and Automation, 2009, pp. 1315-1320, doi: 10.1109/ROBOT.2009.5152522.

[13] M. Ruderman, L. Fridman and P. Pasolli, "Virtual sensing of load forces in hydraulic actuators using second- and higher-order sliding modes", Control Engineering Practice, vol. 92, no. 104151, 2019.

[14] Ali HH, Fales RC, Manring ND (2019) Modeling and control design for an inlet metering valvecontrolled pump used to control actuator velocity via H-infinity and two-degrees-of- freedom methods. https://doi.org/10.1115/1.4044182.

[15] Ali H, Fales R (2020) Robust control design for an inlet metering velocity control system of a linear hydraulic actuator. Int J Fluid Power. https://doi.org/10.13052 /ijfp1439-9776.2113.

[16] Ledezma Pérez, J.,De Pieri, E.,De Negri, V. 2018 September 64. Force Control of Hydraulic Actuators using Additional Hydraulic Compliance. Strojniški vestnik - Journal of Mechanical Engineering. [Online] 64:10.

[17] Ana Lúcia D. Franco, Edson R. De Pieri, Eugênio B. Castelan, Raul Guenther, 2002, DESIGN AND CONTROL OF HYDRAULIC ACTUATORS: SIMULATIONS AND EXPERIMENTAL RESULTS, IFAC Proceedings Volumes, Volume 35, Issue 1, Pp. 19-24, https://doi.org/10.3182/20020721-6ES-1901.01229.

[18] H. H. Ali, A.W. Mustafa, and F.F. Al-Bakri, A new control design and robustness analysis of a variable speed hydrostatic transmission used to control the velocity of a hydraulic cylinder. Int. J. Dynam. Control (2020). https://doi.org/10.1007/s40435-020-00716-w.

[19] Manring ND, Fales RC (2019) Hydraulic control systems. Wiley, Hoboken.

[20] Skogestad, S. and I. Postlethwaite, "Multivariable Feedback Control: Analysis and Design," Second Ed.: John Wiley and Sons, Ltd, 2005. 